C-A/AP/493

October 2013

\title{
Beam-based alignment in RHIC
}

\author{
J. Beebe-Wang
}

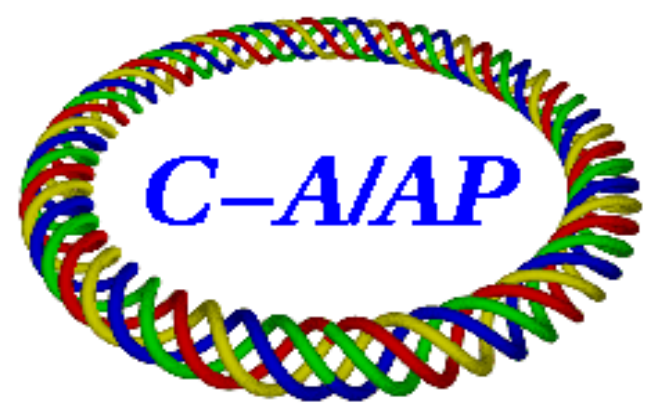

\section{Collider-Accelerator Department Brookhaven National Laboratory Upton, NY 11973}

Notice: This document has been authorized by employees of Brookhaven Science Associates, LLC under Contract No. DE-AC02-98CH10886 with the U.S. Department of Energy. The United States Government retains a nonexclusive, paid-up, irrevocable, world-wide license to publish or reproduce the published form of this document, or allow others to do so, for United States Government purposes. 


\title{
BEAM-BASED ALIGNMENT IN RHIC
}

\author{
Joanne Beebe-Wang \\ Brookhaven National Laboratory, Upton NY, USA
}

\begin{abstract}
A new measurement procedure and a new data analysis algorithm have been introduced to alleviate the difficulties faced by beam-based alignment (BBA) tasks in RHIC. New codes were also developed to implement the new methods in measurements and analysis correspondingly. These improvements have enabled us to successfully perform BBA during the RHIC 2012 and 2013 runs. The BPM offset corrections calculated from the BBA results were implemented and significant improvements were seen in most of the before-and-after comparisons. Here we discuss the measurability and other important BBA issues in RHIC. We present the new measurement procedure, new data analysis algorithm and the new codes that have been developed. We summarize the BBA measurements, analysis, and corrections in RHIC to date. The BBA accuracy limitations are also discussed. As a continuing effort, we also present application code that is under development for future BBA operations in RHIC.
\end{abstract}

\section{BBA Measurability in RHIC}

The standard BBA technique $[1,2]$ in storage rings involves calculating the RMS beam-orbit difference between two states: nominal and slightly modified magnet strength of the quadrupole under investigation with varying beam-toquadrupole offsets. The center of the quadrupole is indicated by the orbit position, read from the beam position monitor (BPM) located near the quadrupole, at which the RMS beam-orbit difference reaches a minimum when a series of beam-toquadrupole offsets are produced by a local orbit bump.

Therefore, in order to perform BBA measurement, a quadrupole has to have: (1) independent control of the power supply; and (2) a nearby BPM with capability of the plane in question. In the RHIC design, not all the quadrupoles satisfy these basic BBA measurement conditions. In this section, we discuss each quadrupole type, 
their power supply control and their relationship to the BPMs. As result of this discussion, we identify all the BBA-measurable quadrupoles in RHIC.

\subsection{Independently Controllable Quadrupoles}

With the RHIC insertion design [3], a series of shunt power supplies is included in the circuit configuration for various insertion quadrupoles ( $Q 9$ and below) in order to control $\beta^{*}$ at the interaction regions (IR). These shunt power supplies make it possible to independently control a number of insertion quadrupoles.

All of the quadrupoles Q1, Q2, and Q3 allow independent control on both sides of an IR. But, none of Q4, Q5, or Q6 can be independently controlled due to the absence of the shunt power supplies.

However, trim quadrupoles TQ4, TQ5, and TQ6 are independently controlled and they may be used to form quasi-pairing with the BPMs associated with Q4, Q5 and Q6. The trim quadrupoles TQ4, TQ5 and TQ6 are located $1.411 \mathrm{~m}, 1.061 \mathrm{~m}$ and $1.061 \mathrm{~m}$ from Q4, Q5 and Q6 respectively. Since these trim quadrupoles are located between the quadrupoles and their associated BPMs, their distance to the BPMs are shorter. The trim quadrupoles TQ4, TQ5 and TQ6 are located $0.618 \mathrm{~m}, 0.601 \mathrm{~m}$ and $0.601 \mathrm{~m}$ from their associated BPMs respectively. The accuracy of the BBA results from these quasi-pairs may not be high, but is expected to be acceptable.

The power supply control of Q7, Q8, and Q9 quadrupoles varies by IR and by the ring. Therefore, their BBA-measurability due to the independent control was individually determined from the insertion quadrupole circuit diagrams [3] (see appendix A).

\subsection{BPM availability for $B B A$}

As described above, only quadrupoles Q1 - Q9 have the possibility of independent control, but not all of those quadrupoles have associated BPMs. For example, all Q2 quadrupoles do not have associated BPMs. Moreover, Q5, Q6, and Q9 have only one plane available. This could be the horizontal or vertical plane depending on location. Quadrupoles Q1, Q3, Q4, Q7, and Q8 are all associated with dual-plane BPMs. This information is summarized in Table 1 and Table 2, along with the availabilities of independent control of power supplies. 


\subsection{BBA-measurable Quadrupoles in RHIC}

Only those quadrupoles with independent control of power supply and a nearby BPM plane are BBA-measureable. In total, taking into consideration power supply constraints from above, there are 250 BPMs with nearby quadrupoles that can benefit from BBA measurements.

The summaries of BBA-able quadrupole/BPM pairs in the Blue and Yellow rings are listed in Table 1 and Table 2, respectively. $P$ stands for independent control of power supply. $B H$ and $B V$ are used to designate which plane, horizontal and/or vertical, is associated with the BPM. The dark shade and light shade indicate unavailabilities of the independent control of power supplies and BPM planes, respectively. The summary of BBA-able quadrupole/BPM pairs in RHIC and the total number of BBA measurements performed on each pair are reported in Section 5.

Table 1: Overview of BBA-able quadrupole/BPM pairs in RHIC's Blue ring.

\begin{tabular}{|c|c|c|c|c|c|c|c|c|c|c|c|c|c|c|c|}
\hline & Q1 & Q2 & Q3 & & Q4 & Q4t & 05 & Q.5t & Q6 & Q6t & & Q7 & & Q8 & $Q 9$ \\
\hline & $\mathrm{PBH} B \mathrm{BV}$ & $\mathrm{P} B \mathrm{BH}$ & \begin{tabular}{|l|l|}
$\mathrm{P}$ & $\mathrm{BH}$ \\
\end{tabular} & BV & $\mathrm{P} B \mathrm{BH} \mathrm{BV}$ & $\mathrm{P} B \mathrm{BHV}$ & $\mathrm{P}|\mathrm{BH}| \mathrm{B}\rangle$ & $\checkmark P B H B V$ & $\mathrm{PBH}$ & P BH BV & & $\mathrm{BH} B \mathrm{BV}$ & $\mathrm{P} B$ & $\mathrm{BH} B \mathrm{BV}$ & $\mathrm{PBH} B \mathrm{BV}$ \\
\hline bi1 & & & & & & & & & & & & & J & & \\
\hline bo2 & & & & & & & & & & & & & & & \\
\hline bo3 & & & & & & & & & & & & & & & \\
\hline bi4 & & & & & & & & & & & & & & & \\
\hline bi5 & & & & & & & & & & & & & & & \\
\hline bo6 & & & & & & & & & & & & & & & \\
\hline bo7 & & & & & & & & & & & & & & & \\
\hline bi8 & & & & & & & & & & & & & & & \\
\hline bi9 & & & & & & & & & & & & & & & \\
\hline bo10 & & & & & & & & & & & & & & & \\
\hline bo11 & & & & & & & & & & & & & & & \\
\hline bi12 & & & & & & & & & & & & & & & \\
\hline
\end{tabular}


Table 2: Overview of BBA-able quadrupole/BPM pairs in RHIC's Yellow ring.

\begin{tabular}{|c|c|c|c|c|c|c|c|c|c|c|c|c|}
\hline & Q1 & Q2 & Q3 & Q4 & Q4t & Q5 & Q5t & Q6 & Q6t & Q7 & Q8 & Q9 \\
\hline & $\mathrm{P}|\mathrm{BH}| \mathrm{BV}$ & $\mathrm{P}|\mathrm{BH}| \mathrm{BV}$ & $\mathrm{P} B \mathrm{BH} \mid \mathrm{BV}$ & $\mathrm{P} B \mathrm{BH}$ & $\mathrm{P} B \mathrm{BH}$ & $\mathrm{P} B \mathrm{BH}$ & $\mathrm{P}|\mathrm{BH}| \mathrm{BV}$ & $|\mathrm{P}| \mathrm{BH} \mid \mathrm{B}$ & $/ \mathrm{P} B \mathrm{BH}$ & $\mathrm{P}|\mathrm{BH}| \mathrm{B}$ & $\mathrm{V} / \mathrm{P} B \mathrm{BH}$ & $\mathrm{P} B \mathrm{BH}$ \\
\hline yo1 & & & & & & & & & & & & \\
\hline yi2 & & & & & & & & & & & & \\
\hline yi3 & & & & & & & & & & & & \\
\hline yo4 & & & & & & & & & & & & \\
\hline yo5 & & & & & & & & & & & & \\
\hline yi6 & & & & & & & & & & & & \\
\hline yi7 & & & & & & & & & & & & \\
\hline yo8 & & & & & & & & & & & & \\
\hline yog & & & & & & & & & & & & \\
\hline yi10 & & & & & & & & & & & & \\
\hline yi11 & & & & & & & & & & & & \\
\hline yo12 & & & & & & & & & & & & \\
\hline
\end{tabular}

\section{Important Issues regarding BBA in RHIC}

BBA measurement is difficult in RHIC for the following reasons:

1. There are very limited dipole correctors, especially in the Interaction Region. For example, in order to make measurements on the triplet magnets one needs to use the BBA bump (3-corrector bump) that includes not only the triplet quadrupoles, but also 3-4 additional quadrupoles, 2 DX magnets and 2 D0 magnets.

2. The effects of quadrupoles Q1, Q2, Q3 are equally strong and they cannot be decoupled.

3. There are magnet alignment errors.

4. The beam angles at the quadrupoles are unknown due to the unknown beam positions and angles at the first corrector of the BBA bump.

5. The RHIC rings are very large. When all the BPMs in all the arcs are used, some BPMs contribute to noise more than signal.

6. The signal to noise ratio is low. 
In this section we concentrate on the following three issues since they are crucial to the BBA in RHIC.

\subsection{RHIC Insertion Quadrupole Excitations}

Each RHIC ring consists of 6 insertions joining the 3 inner arcs and 3 outer arcs. Each insertion has 9 quadrupoles and 6 dipoles ( 4 for dispersion matching and 2 for the beam crossing) on each side of the crossing point [3]. Each half-insertion is composed of (1) a dispersion matching section (D9, Q9, D8, Q8, Q7, D6, Q6 and D5); (2) a straight betatron function matching section with the quadrupole doublet Q5, Q4 and the triplet Q3, Q2 and Q1; and (3) the beam crossing dipoles D0 and DX.

There is near perfect reflection anti-symmetry relative to the crossing point in component location and component strength. A small deviation from symmetry is caused by the differences in drift space lengths imposed by geometrical constraints and the differences between QF and QD $\left(\sim 2 \times 10^{-3}\right)$. The inner and outer halfinsertion lengths are $156.523 \mathrm{~m}$ and $156.608 \mathrm{~m}$, respectively. This symmetry breaking results in arc beta and dispersion function flutter of $<5 \times 10^{-3}$ when the insertion quadrupoles are excited with exact anti-symmetry. A fully matched lattice, i.e. without lattice function flutter in the arcs, can be obtained by allowing differences in the inner/outer quadrupole excitations of $\sim 10^{-2}$.

\subsection{RHIC BPM offsets and surveys}

RHIC was designed with 288 "CQS packages", which are comprised of corrector-quadrupole-sextupole and optionally a BPM in a rigid mechanical assembly. The CQS packages were surveyed "on the bench" before installation whereby any BPM-to-reference orbit and magnet-to-BPM offsets were recorded [3]. The latter of these measurements are used in the RHIC controls system to calibrate BPM alignment with respect to quadrupole centers. Installation tolerances for quadrupoleBPM misalignment were RMS $\Delta \mathrm{x}_{\mathrm{rms}}=\Delta \mathrm{y}_{\mathrm{rms}}=0.25 \mathrm{~mm}$.

The CQS packages are designed with leads for the magnets and instrumentation at only one end and are installed to minimize the amount of superconducting buswork and penetrations through the cryostat. This consideration is independent of beam direction, so the orientation of the packages varies throughout the ring depending on where the nearest cold cryostat is. Equal numbers 
of packages were installed with each of the two possible orientations. Therefore, of the 288 packages containing BPMs, 144 have an orientation of -1 , defined here as the orientation in which the lead end of the package is the downstream end. This orientation must be used when applying offsets if accurate beam positions are to be reported.

When the BPM offsets were first introduced into the RHIC control system, the orientation of each CQS package was not properly taken into consideration. The result of this oversight caused a systematic error in BPM offsets with twice the magnitude of the survey value (the implemented offset being the opposite sign of, but equal in magnitude to, the correct setting) in the 144 packages installed with -1 polarity.

To further complicate the issue, the BPM survey offsets provided were incorrectly interpreted or implemented by a factor of -1 . This was in addition to and independent of CQS orientation and therefore affected vertical plane BPMs as well as horizontal. The result of these two issues working in concert was a systematic offset error in all vertical and roughly half the horizontal BPMs.

BBA measurements at the first part of run 10 were devoted to the verification of these offset reversals and to the related corrections. Therefore, this report includes the BBA works after Jan. 21, 2010 when the BBA offset reversal corrections were completed.

\subsection{BPM Accuracy and Beam Position Acquisition}

In addition to the BPM location in reference to quadrupoles as discussed above, the BBA accuracy is also governed by these important factors in the BPMs: resolution, accuracy and timing; beam position acquisition and calculations of average orbit used by BBA measurements.

BPM accuracy places a fundamental limit on the accuracy of BBA, though this limit is rarely reached. BPM resolution is about $10 \mathrm{~mm}$ for a constant temperature, but at present, each 10 degree temperature change results in $\sim 1$ ns change in trigger time which adds an uncertainty on the order of hundreds of microns up to $1 \mathrm{~mm}$ on a $B P M$ reading. In an effort to combat this effect, when possible, the BPM timing was calibrated by the experts immediately prior to the data taking.

Since run 2009, a new continuous average orbit calculation was implemented

in the RHIC BPM system. Average orbits are computed by adding a percentage of each new position measurement to the average orbit position value. The result of 
calculating average orbits in this way is that a step in beam position requires several iterations of the calculation before the value settles.

This effect must be taken into consideration when designing a measurement algorithm for BBA. The BPM trigger mode is set to $1 \mathrm{HZ}$ throughout a BBA measurement so it is important to remain at each bump size long enough for readings to settle. Figure 1 shows a comparison between the average orbit data (red) recorded once every second and the data (black) from $10 \mathrm{~Hz}-\mathrm{BPM}$ electronics recorded at $1 \mathrm{KHz}$ on a typical BPM (bo6-bh3). It is clear, from Figure 1 , that the time needed for readings to settle is of the order of several seconds and depends on the relative bump size. Recent BBA measurements have implemented a 5 second wait time for bumps $2 \mathrm{~mm}$ or larger and a 3 second wait time otherwise.

The overall accuracy of the BBA procedure will be discussed in more detail in Section 6.

May 2, 2012 BBA on bo6-bh3

Comparison of $10 \mathrm{~Hz}$ and Average Orbit Data

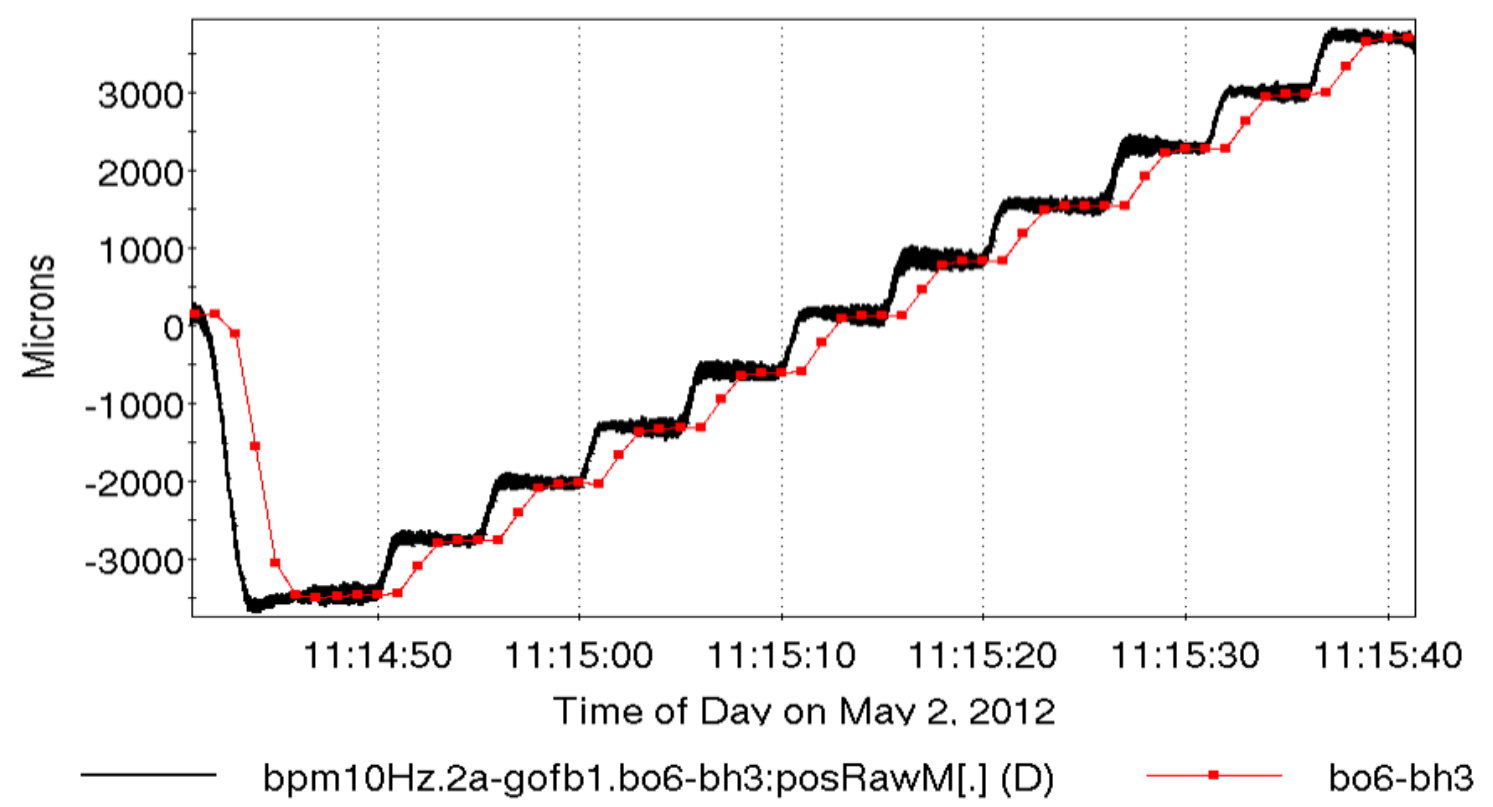

Figure 1: A comparison of BPM response time between the average orbits calculation used in BBA (red) recorded once every second and the data (black) from $10 \mathrm{~Hz}-\mathrm{BPM}$ electronics recorded at $1 \mathrm{KHz}$ on the same BPM. 


\section{Pre-2012 BBA Measurements and Results}

The first part of the BBA activity during run 2010 was dedicated to verifying the RHIC BPM offset reversals as described in subsection 2.2. During the remainder of run 2010, 20 sets of BBA measurement data were taken on the Blue IR $6 \& 8$ and 30 sets were taken on the Yellow IR 2. All the raw data are shown in Figure 2. The measurements were made on Au beams at injection and yield average error bars of $216 \mathrm{~mm}$ and $153 \mathrm{~mm}$ in the Blue and Yellow rings, respectively.

In total, 27 sets of measurements were taken during run 2011. The raw data are shown in Figure 3, where 24 sets were taken in the Blue ring and and 3 sets in the Yellow ring, with average error bars of $310 \mathrm{~mm}$ and $352 \mathrm{~mm}$ respectively.

The raw data collected during the 2010 and 2011 runs was limited due to excessive beam loss, noise and a limited analysis algorithm. Accordingly, all the pre2012 data were re-evaluated and re-analyzed with a new algorithm during run 2012. 77 sets in total ( 44 sets in the Blue ring, 33 sets in the Yellow ring) were identified to be useful and were utilized to aid the 2012 and 2013 BBA activities. The error bars of the processed BBA results were found to be $267 \mathrm{~mm}$ and $171 \mathrm{~mm}$ in the Blue and Yellow rings, respectively. Further details on the pre-2012 runs can be found in references $[4,5]$. 


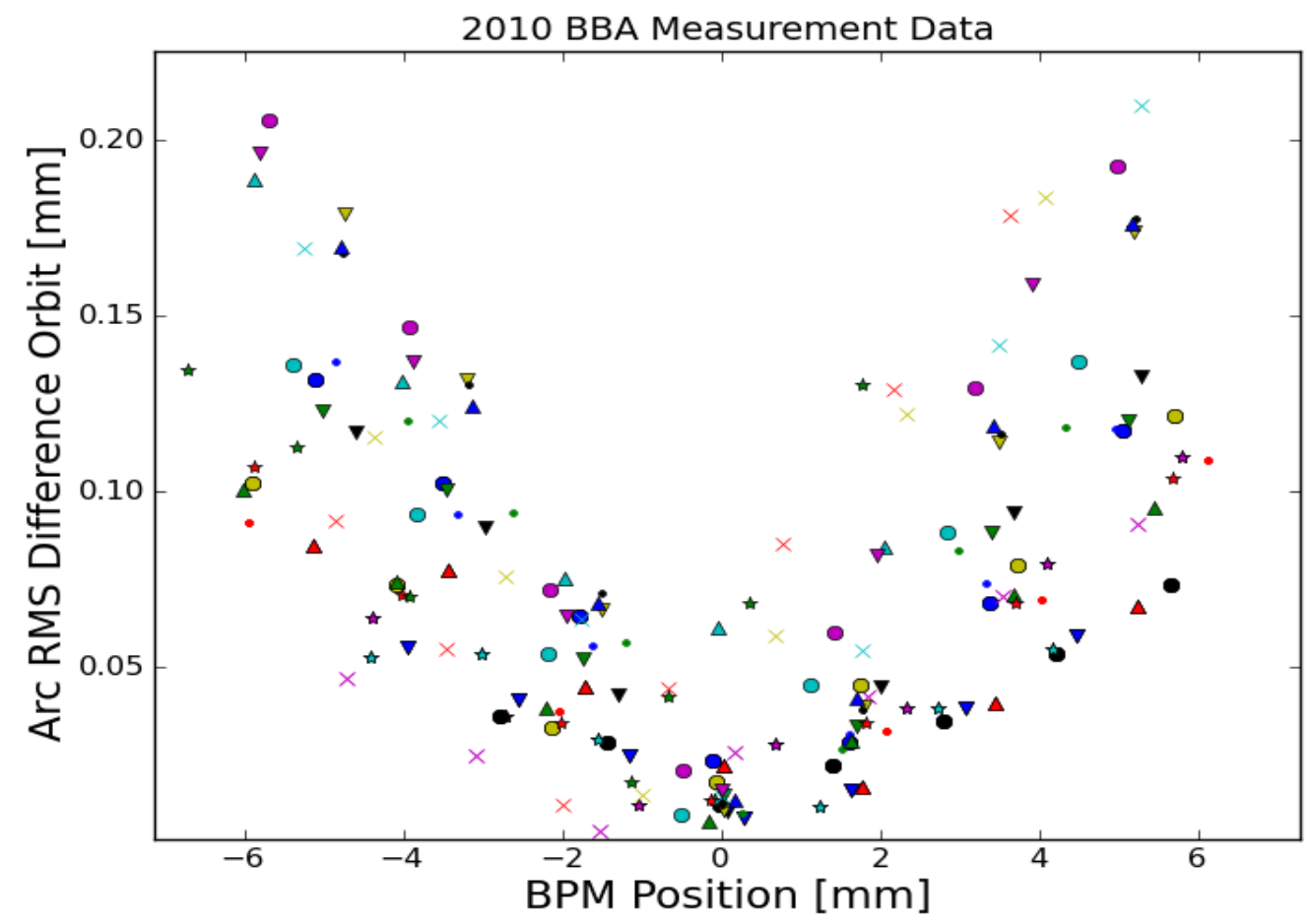

Figure 2: Superimposed BBA measurement data obtained during run 10.

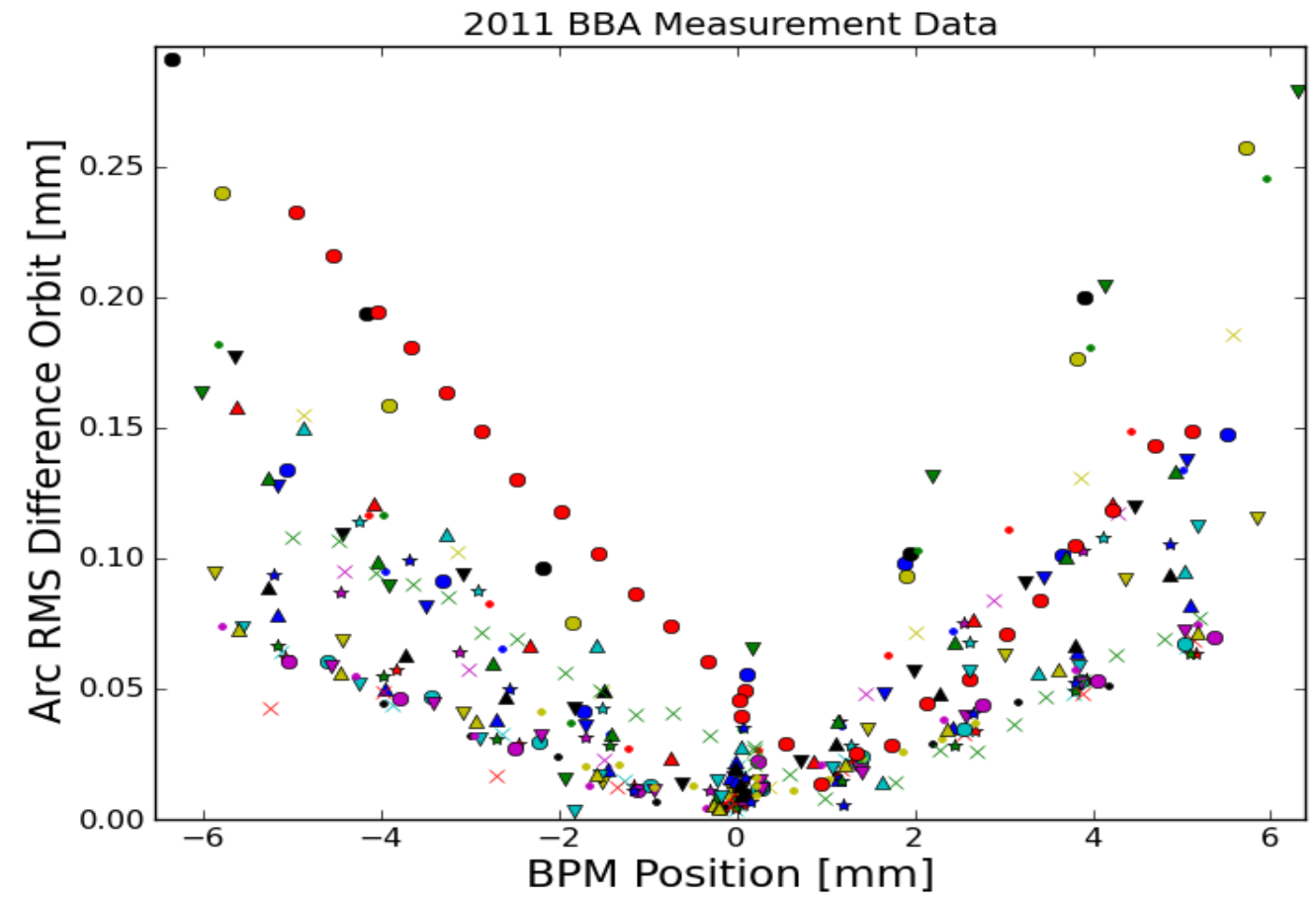

Figure 3: Superimposed BBA measurement data obtained during run 11. 


\section{Improvements Made During Run 2012-2013}

\subsection{New Measurement Procedure}

A major effort was made during the 2012 BBA activity to explore possible solutions to the challenges faced on RHIC. One issue was the unacceptably low level of signal to noise ratio. This was addressed by carefully investigating the raw BBA measurement data obtained during runs 2010 and 2011, and introducing two major changes into the BBA measurements: (1) a new measurement algorithm which decreases the noise; (2) optimization of the measurement parameters, which increases the signal. These two changes brought the signal to noise ratio to an acceptable level for the remaining and any future measurements of BBA in RHIC.

In the traditional BBA measurement procedure the beam was steered stepby-step across the central region of the quadrupole in either the horizontal or vertical direction by a three-corrector-bump. At each beam position the quadrupole strength was modulated, then returned to the initial state. As an example, Figure 4 shows the power supply current of dipole corrector bo7-th2 (light blue line) during BBA measurement of quadrupole bi8-qf3 using the traditional procedure with 11 positionsteps. The power supply current of quadrupole bi8-qf3 (dark blue line) was changed 22 times (modulated 11 times and returned to the initial state after each modulation), which is very typical of traditional measurement procedures. It was found from careful investigation that the noise in the measurement could be considerably reduced by minimizing the frequency of the changes in quadrupole strength during the BBA measurement. To this end, we developed a new BBA measurement algorithm named " $1 \mathrm{Q}$ ", in which the quadrupole strength is modulated only once, and returns to the initial state once, during the entire procedure. As an example, Figure 5 shows how the currents change with the new algorithm applied to the same power supplies as in Figure 4. In the new procedure, the power supply current of quadrupole bi8-qf3 (dark blue line) was modulated and returned to the initial state. Before and after this modulation, the current of dipole corrector bo7-th2 (light blue line) was changed 12 times during two identical 12-steps beam position scans. 
BBA Power Supplies 03/15/2011

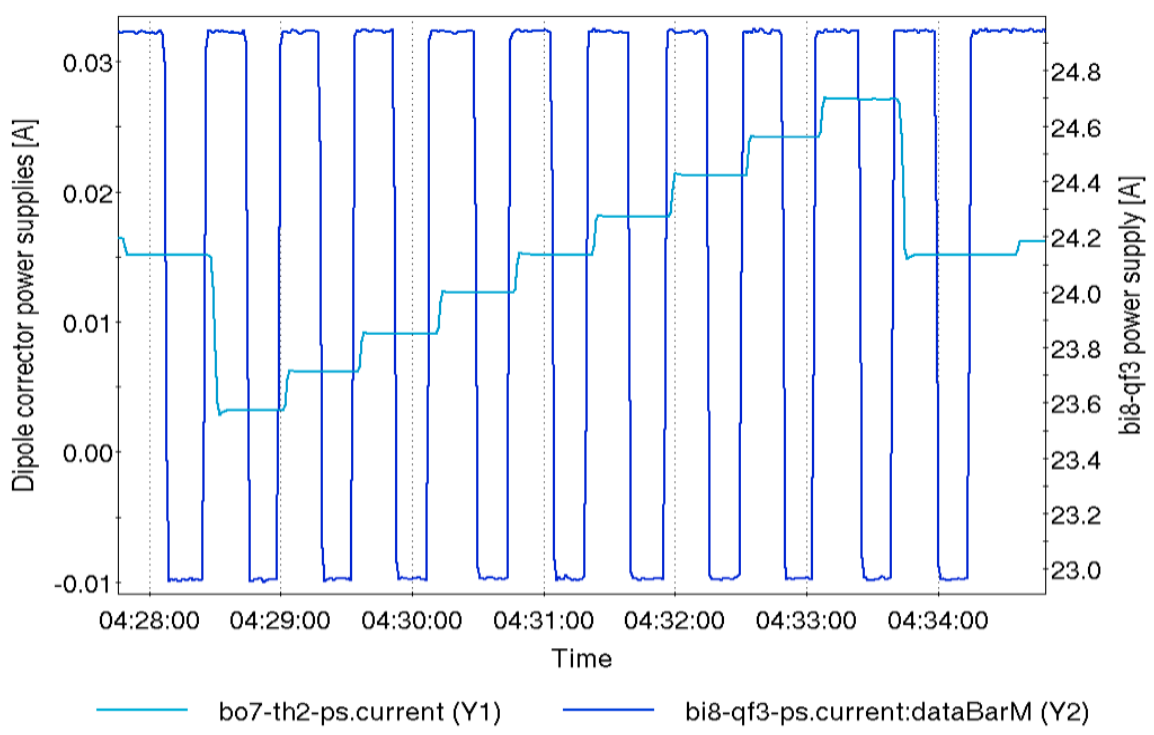

Figure 4: The typical BBA procedure before run 12 involved modulating a quadrupole once (two movements) for each position step. The figure shows the power supply currents for one of the correctors and the quadrupole involved in a measurement from run 2011.

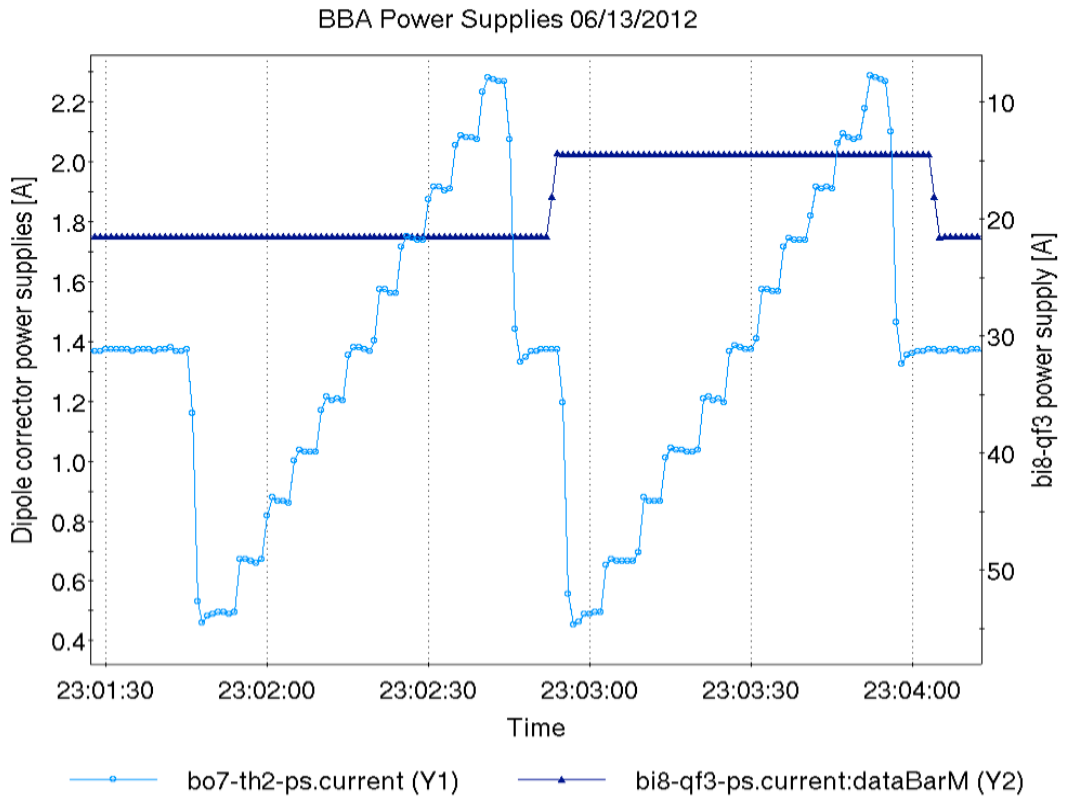

Figure 5: New BBA measurement procedure implemented for run 2012 and 2013. Above are the same power supplies used in Figure 4, but from a measurement in run 2012. 
It was also found that a careful choice of beam-offset range and the amount of quadrupole strength change in a given lattice can improve the signal of the measurements without much sacrifice of beam lost. For example, the best measurement parameters at injection are a beam-offset range of [-5.0 5.0] $\mathrm{mm}$ in conjunction with quadrupole strength change of -0.002 for most of Q1s and Q3s. Figure 6 compares BBA measurement data obtained during runs 2010, 2011 and 2012. It can be observed that the signal (vertical scale) is much stronger in the 2012 BBA data (light blue) compared to the BBA data of run 2010 (dark blue) and run 2011 (red).

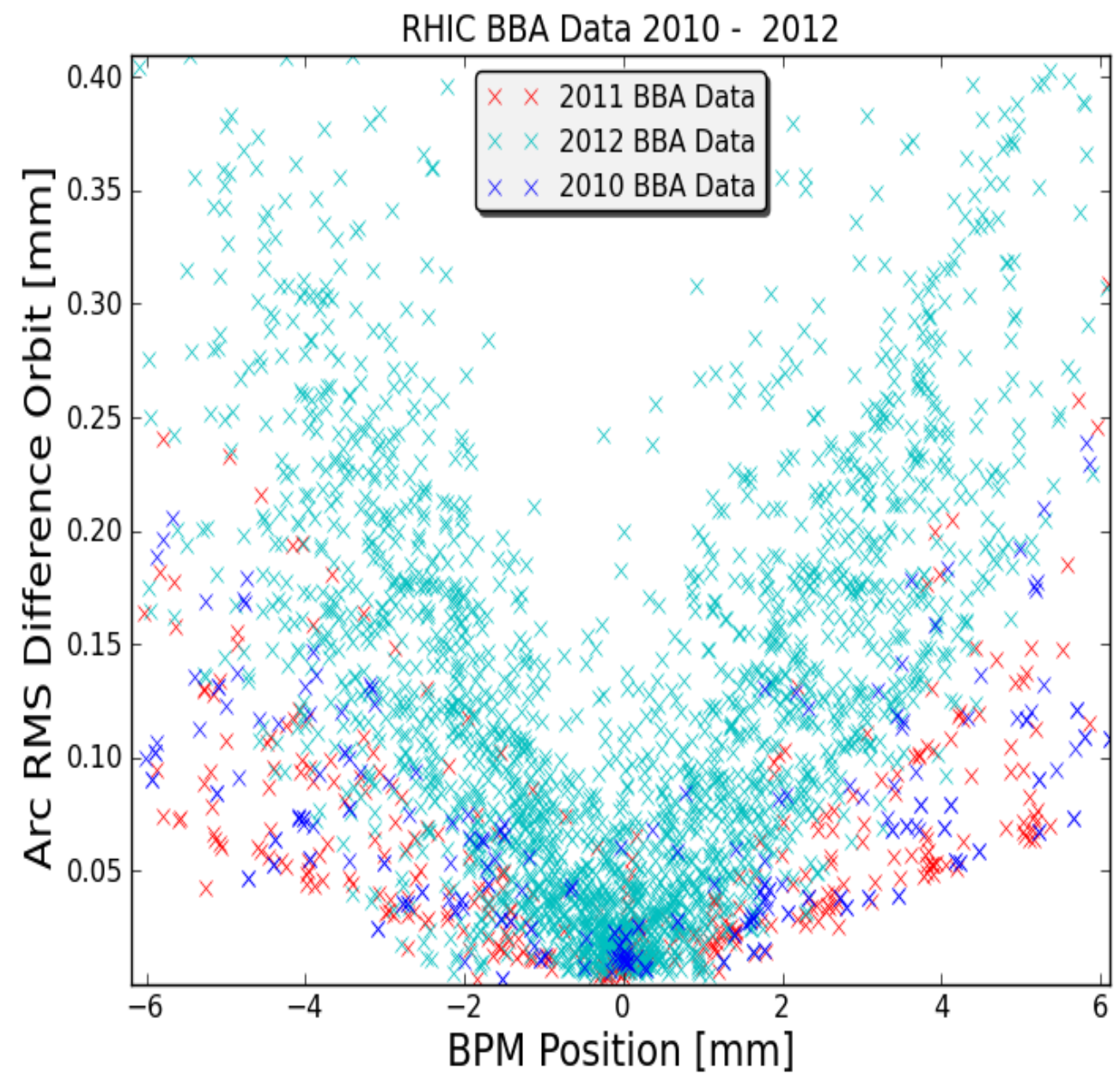

Figure 6. Comparison of BBA measurement data obtained during runs 2010, 2011 and 2012. 


\subsection{New Data Analysis Algorithm}

Based on the physical model of the fields in the quadrupole magnets and on the statistical characteristics of the signals, a sophisticated data analysis algorithm was developed. Figure 7 shows the flowchart of the new BBA algorithm.

The error bar calculations include three sources: (1) RMS values of raw data fitted to a linear model for the magnetic fields on both sides of the quadrupoles; (2) Stability of the beam position during the time period when the BBA data was collected; (3) Repeatability of the data points with the same machine settings and the same measurement parameters.

This algorithm was used to analysis all the measurement data obtained during runs 2012 and 2013, as well as to re-analysis pre-2012 measurement data.

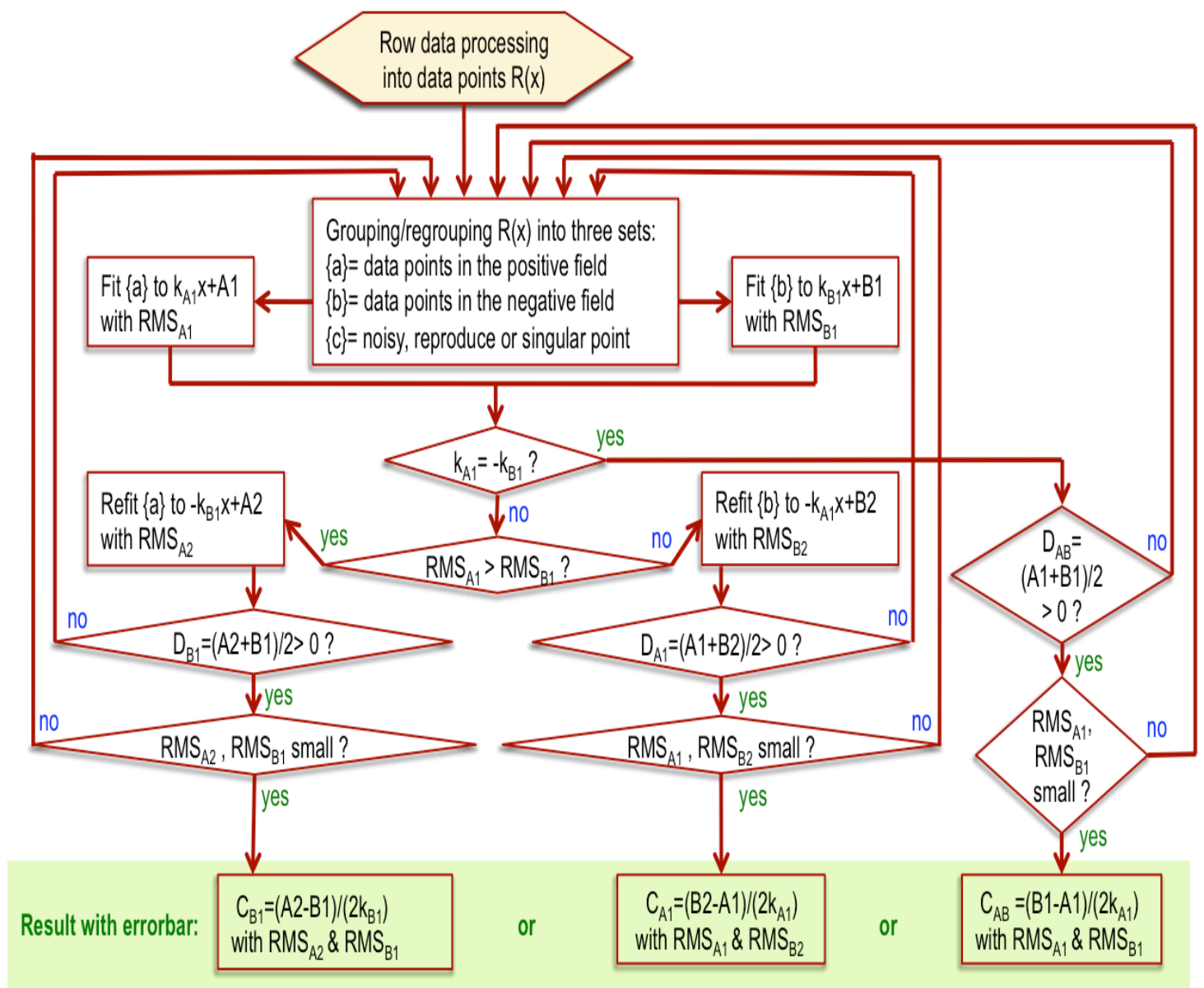

Figure 7: The flowchart of the new data analysis algorithm based on the physical model. 


\subsection{Code development for data acquisition and analysis}

There have been two major achievements as a result of the recent BBA code development. One was consolidating measurement and analysis into two easy-touse and dependable scripts before and during run 2012. The other is the fully operational BBA application under construction in 2013.

During RHIC's 2010 and 2011 runs, a set of bash, gnuplot, and perl scripts were used for measurement and analysis. The orbit bumps had to be built manually after looking up the proper coefficients for each power supply involved. This was very time consuming and error prone.

The data acquisition code and data analysis code were completely rewritten for the RHIC 2012 run and thereafter. Python was chosen for a number of reasons including readability, integration of matplotlib, and the ability to use modules already developed for interacting with a newly created bump manager for RHIC. The new bump manager allows the user to simply supply the parameters for a bump without having to construct bump coefficients manually.

After the successful changes made during run 2012, the BBA procedure has become stable enough to be developed further to include a GUI, to combine measurement and analysis, and to document previous measurements, all from the same application. This will move the procedure from a script-based, expert-only activity to one available to a wider community. It will also allow for installation of offsets directly from within the application.

Work is being done to build a $\mathrm{C}++$ application with the same look, feel, and operation that is standard to the C-AD controls system. It has been tested and debugged during run 2013 and will hopefully be fully operational for run 2014.

\section{BBA Measurements and Results in Run 2012-2013}

The new measurement procedure and the analysis have been highly successful during the RHIC runs 2012 and 2013.

For the first time in RHIC's history, a complete set of BBA data of triplet quadrupoles (Q1 and $\mathrm{Q} 3$ ) in all interaction regions was obtained in both the 
horizontal and vertical planes. BBA data of trim-quadrupole-planes, which had never been collected before, has also been explored and analyzed during run 2012. These experiences have served as the foundation for the systematic measurements on all the BBA-able quadrupole/BPM pairs in RHIC during run 2013. In total 128 sets of useful raw data were collected (60 sets in the Blue ring, 68 sets in the Yellow ring) during the RHIC 2012 run. All the raw data are shown in Figure 8. The average error bar was $138 \mathrm{~mm}$.

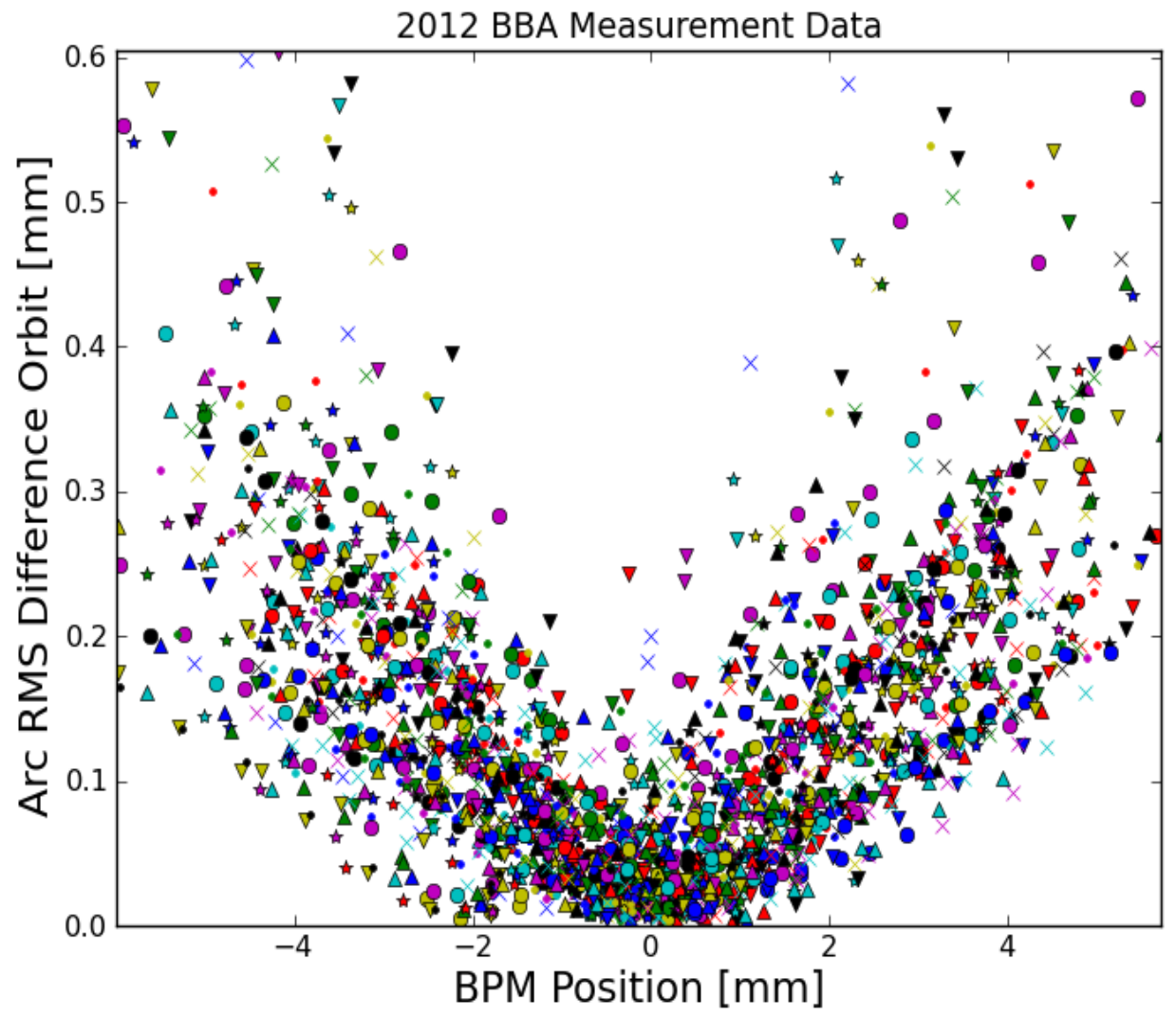

Figure 8: Superimposed BBA measurement data obtained during RHIC 2012 run.

All the BPM offset corrections calculated from the BBA results have been implemented during and at end of RHIC's 2012 run. In order to confirm the improvement after the corrections, some quadrupole/BPM pairs that obtained large BPM offset corrections were subjected to re-measurement and re-analysis. Figures 9 and 10 show the results before and after the BBA corrections in the Blue and Yellow rings, respectively. 

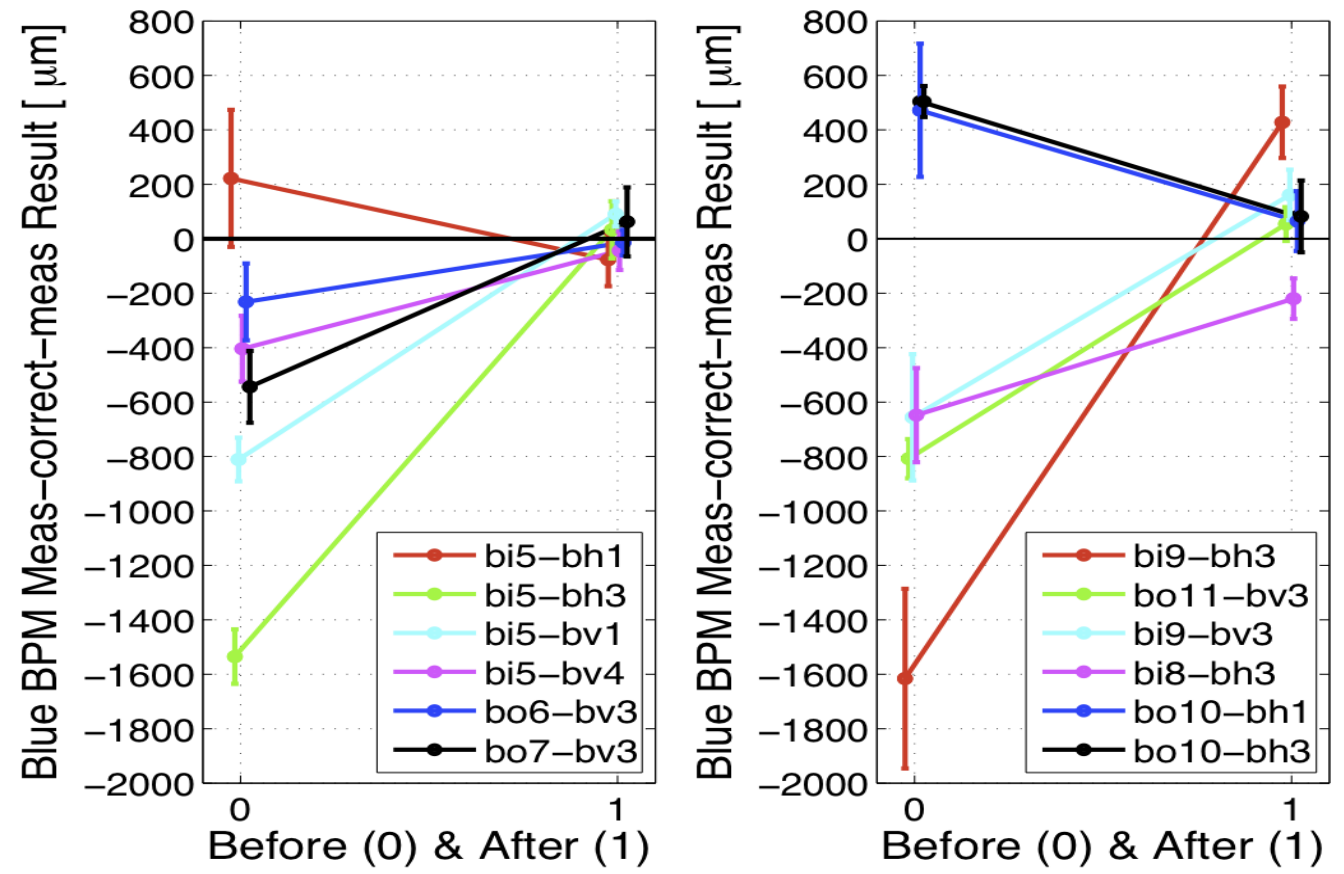

Figure 9: Measurement results before and after the BBA corrections in the Blue ring during RHIC 2012 run.
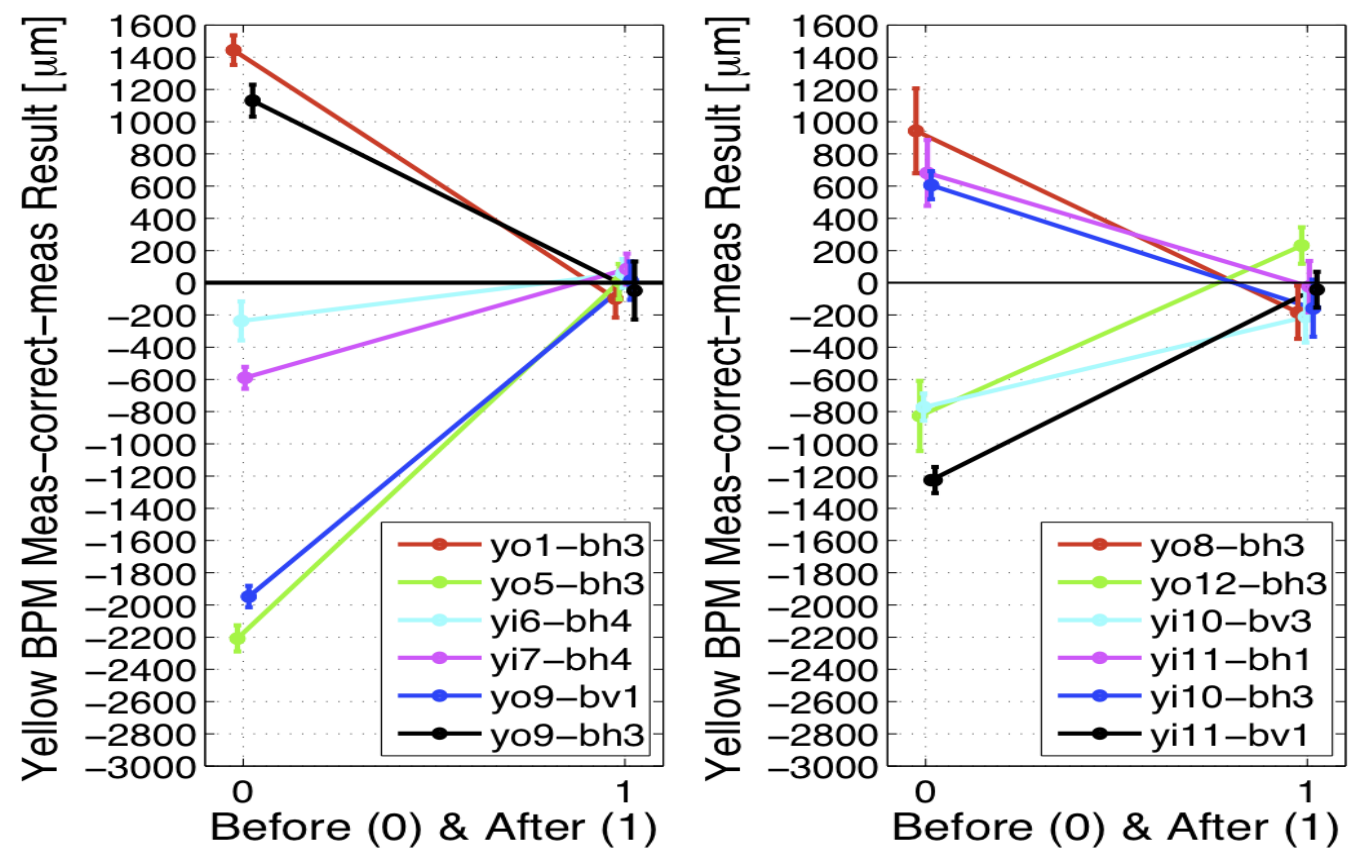

Figure 10: Measurement results before and after the BBA corrections in the Yellow ring during RHIC 2012 run. 
The BBA activity during the RHIC's 2013 run was concentrated on collecting the BBA data on all the BBA-measurable quadrupole/BPM pairs that had never been collected before. Details of this activity in the Blue and Yellow rings are summarized in Tables 3 and 4, respectively. The black and red numbers indicate the total number of measurements obtained before and during run 2013, respectively. The gray shade indicates un-BBA-able quadrupole/BPM pairs. The green shade indicates the quadrupole/BPM pairs that can not be measured during polarized proton runs due to the snake bumps, while the yellow shade indicates measurements that were attempted but not obtained due to other technical difficulties. Figure 11 shows results before and after the BBA corrections re-measured during RHIC's 2013 run.

Table 3: Summary of total number of measurements on each BBA-able quadrupole/BPM pairs in RHIC's Blue ring obtained before (black numbers) and during run 2013 (red numbers).

\begin{tabular}{|c|c|c|c|c|c|c|c|c|c|c|c|c|c|c|c|c|}
\hline & $\overline{b h 1}$ & $\overline{b v 1}$ & bh3 & bv3 & bh4t & bv4t & bh5t & bv5t & bh6t & bv6t & bh7 & bv7 & bh8 & bv8 & bh9 & bv9 \\
\hline bi1- & $2+1$ & 3 & 2 & 1 & 3 & 2 & 2 & & & 1 & & & & & 2 & \\
\hline bo2- & $1+1$ & 2 & 1 & 1 & 2 & 1 & & 2 & & & & & 2 & & & \\
\hline bo3- & $1+1$ & 1 & 1 & 1 & 1 & 1 & & 2 & 2 & & & & 2 & & & \\
\hline bi4- & 1 & 1 & 1 & 1 & 1 & 1 & 2 & & & 2 & 1 & & & & 2 & \\
\hline bi5- & $7+1$ & $3+1$ & 4 & 5 & 2 & 2 & 7 & & & 3 & & & & & 2 & \\
\hline bo6- & $3+1$ & $3+2$ & 3 & 3 & $1+1$ & $1+1$ & & 2 & 2 & & & & 2 & & & \\
\hline bo7- & $2+1$ & 2 & 4 & 4 & 1 & 1 & & 3 & 1 & & & & 2 & & & \\
\hline bi8- & $9+1$ & 3 & $5+1$ & 3 & $1+1$ & $1+1$ & 2 & & & 2 & & & & & 2 & \\
\hline bi9- & $1+1$ & 1 & 2 & 2 & 1 & & 2 & & & & & & & & 2 & \\
\hline bo10- & $2+1$ & 2 & 2 & 1 & & 1 & & 2 & 2 & & & & 2 & & & 2 \\
\hline bo11- & $1+1$ & 1 & 1 & 2 & 1 & 1 & & 2 & 1 & & & & 2 & & & \\
\hline bi12- & $1+1$ & 1 & 1 & 2 & 1 & 1 & 2 & & & 2 & & & & & 2 & \\
\hline
\end{tabular}


Table 4: Summary of total number of measurements on each BBA-able quadrupole/BPM pairs in RHIC's Yellow ring obtained before (black numbers) and during run 2013 (red numbers).

\begin{tabular}{|l|r|r|r|r|r|r|r|r|r|r|r|r|r|r|r|r|}
\hline & bh1 & bv1 & bh3 & bv3 & bh4t & bv4t & bh5t & bv5t & bh6t & bv6t & bh7 & bv7 & bh8 & bv8 & bh9 & bv9 \\
\hline yo1- & 3 & 2 & 3 & 2 & 1 & 1 & & 2 & 2 & & & & 1 & & & \\
\hline yi2- & 2 & 1 & 2 & 4 & 1 & 1 & 2 & & & 2 & & & & & 1 & \\
\hline yi3- & 1 & 1 & 1 & 3 & 1 & 1 & 3 & & & & & & & & 2 & \\
\hline yo4- & 1 & 1 & 1 & 1 & 1 & 1 & & 4 & 3 & & 2 & & 2 & & & \\
\hline y05- & $9+1$ & 3 & 11 & 2 & 1 & 1 & & 2 & 2 & & & & 2 & & & \\
\hline yi6- & $5+1$ & 3 & 4 & 1 & 2 & 1 & 2 & & & 3 & & & & & 4 \\
\hline yi7- & 5 & 1 & 3 & 2 & 1 & 1 & 1 & & & 2 & & & & & 2 & \\
\hline yo8- & 4 & 2 & 8 & 2 & $1+1$ & 1 & & 2 & 1 & & & & 3 & & & \\
\hline yo9- & 1 & 2 & 3 & 1 & & 1 & & 3 & 2 & & & & 1 & & & \\
\hline yi10- & $1+1$ & 1 & 2 & 2 & 1 & 1 & 2 & & & 2 & & & & 2 & 1 & \\
\hline yi11- & 2 & 2 & 1 & 1 & 1 & 1 & 3 & & & 2 & & & & & 2 & \\
\hline yo12- & 1 & 1 & 2 & 1 & 1 & 1 & & 3 & 2 & & & & 2 & & & \\
\hline
\end{tabular}
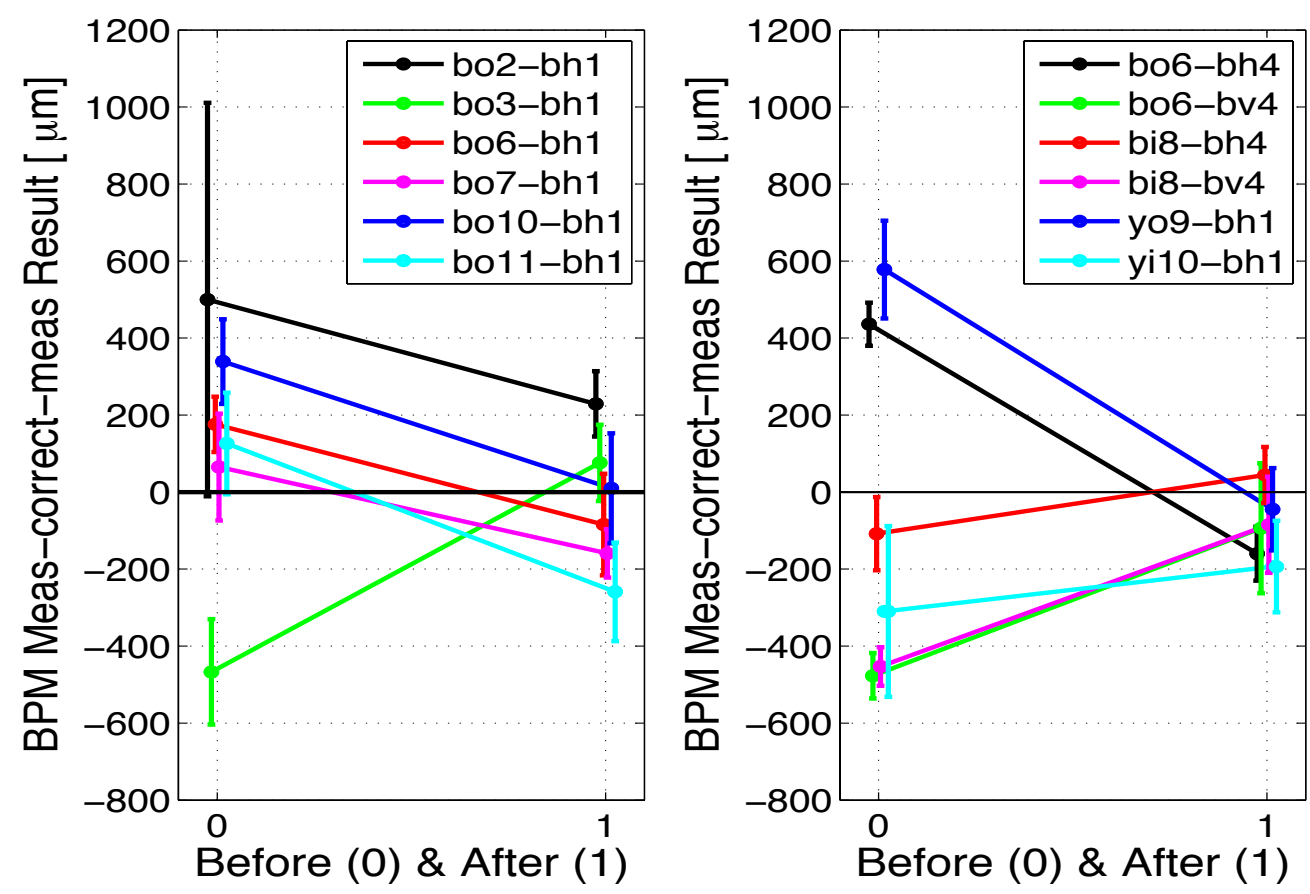

Figure 11: Measurement results before and after the BBA corrections in the Blue and Yellow ring during RHIC 2013 run. 


\section{BBA Accuracy Limitation on RHIC}

The accuracy limitation of BBA on RHIC is about $0.1 \mathrm{~mm}$ because of the following factors:

1. BPM accuracy is temperature dependent. Each $\Delta 10^{\circ} \mathrm{F}$ temperature change results in $\sim \Delta 1$ ns change in trigger time which adds an uncertainty of $\sim+/-$ $0.1 \mathrm{~mm}$ to $\sim 1 \mathrm{~mm}$ in BPM reading. BPM resolution is $\sim 0.01 \mathrm{~mm}$ at a constant temperature;

2. The physical misalignment of quadrupoles relative to each other is $\sim+/-$ $0.06 \mathrm{~mm}$;

3. BPM position with respect to the outside fiducials is $\sim+/-0.13 \mathrm{~mm}$;

4. Unknown beam positions and angles at the first corrector (beam angle at the triplet). It was estimated to have an effect $\sim+/-0.1 \mathrm{~mm}$;

5. Repeatability under the same machine settings is found by experiment to be $\sim+/-0.1 \mathrm{~mm}$.

\section{BBA Documentation and BPM Offset Correction Installation}

In order to improve the BBA accuracy, one needs to know the conditions and the parameters of all the elements involved in performing each measurement. Evaluation of the BPM offset corrections resulting from data processing and analysis needs to be based on all known factors, including especially the comparison of multiple measurements on the same quadrupole. Importantly, clear documentation has to be maintained of all BBA activation in RHIC, and to ensure this was the case, the following methods were utilized during run 2012 and run 2013:

1. Two database tables were regularly updated. The "BBAelem" database contains the complete set of beamline elements including quadrupoles, BPMs and dipole correctors (384 in total) with 15 searchable attributes. In addition, 
the "BBAMeas" database contains all the BBA measurements made on RHIC to date (457 sets) with 37 searchable attributes. Both tables are accessible online (http://www.cadops.bnl.gov/Controls/apps/dbbrowser/).

2. BBA elog records were routinely kept: a) Pre-measurement planning, notes during measurement sessions, post measurement summaries; b) Data processing at preliminary and final stages, with graphs and results; c) BBA related discussions and communications. The log is accessible online (http://www.cadops.bnl.gov/cgi-bin/elog/).

3. Run-time log-files are saved automatically by the measurement script.

4. IPAC 2013 paper [6] reports the 2012 activities and major results. PAC 2011 paper [5] reported the 2010 and 2011 activities and major results.

The adjustable electronic BPM offset parameter, called orbPositionOffsetS on RHIC, is the parameter that is adjusted when BBA results are committed. orbPositionOffsetS is added to the raw BPM calculation before being reported to the controls system. Changing the value itself is straightforward, but depending on when these changes are made, a number of other considerations must be made.

In order to have the least possible impact on operations, the offsets were installed at the end of physics stores when their effects could best be studied. Because of RHIC's current dependence on periodic orbit feedback, it is important to install offsets at the correct time. The procedure is as follows. Note that capturing a new goal orbit immediately after offset installation ensures that nothing physically changes the orbit -- only the readings on the BPMs change.

1. Trigger a $1 \mathrm{~Hz}$ average orbit event -- the standard is $4 \mathrm{~Hz}$ during a store

2. Turn off the $10 \mathrm{~Hz}$ feedback if installing to a $10 \mathrm{~Hz}$ BPM

3. Disable store orbit corrections

4. Optimize via LISA if necessary -- this is highly dependent on the running state

5. Install desired offsets with BBA offset installation script prepared in advance

6. Capture a new goal orbit in the affected rings

7. Turn on the $10 \mathrm{~Hz}$ feedback and enable store orbit corrections

8. Check orbits, collision rates, document results. 


\section{Summary and Conclusion}

As a result of this work, RHIC's BBA is generally producing reliable results. It takes 3-4 min for each set of measurements with good accuracy/resolution. Measurements at Q1 \& Q3 have been completed in both rings (24H, 24V each ring). For the first time in RHIC's history, all the BBA-measurable quadrupole/BPM pairs in RHIC are identified and the measurements on most of them have been attempted. Offset corrections have been installed and the results are generally good.

The BBA codes have been significantly improved to increase the usability and reliability. In addition, a stand-alone GUI application is currently under development for future BBA operations in RHIC. 


\section{Appendix}

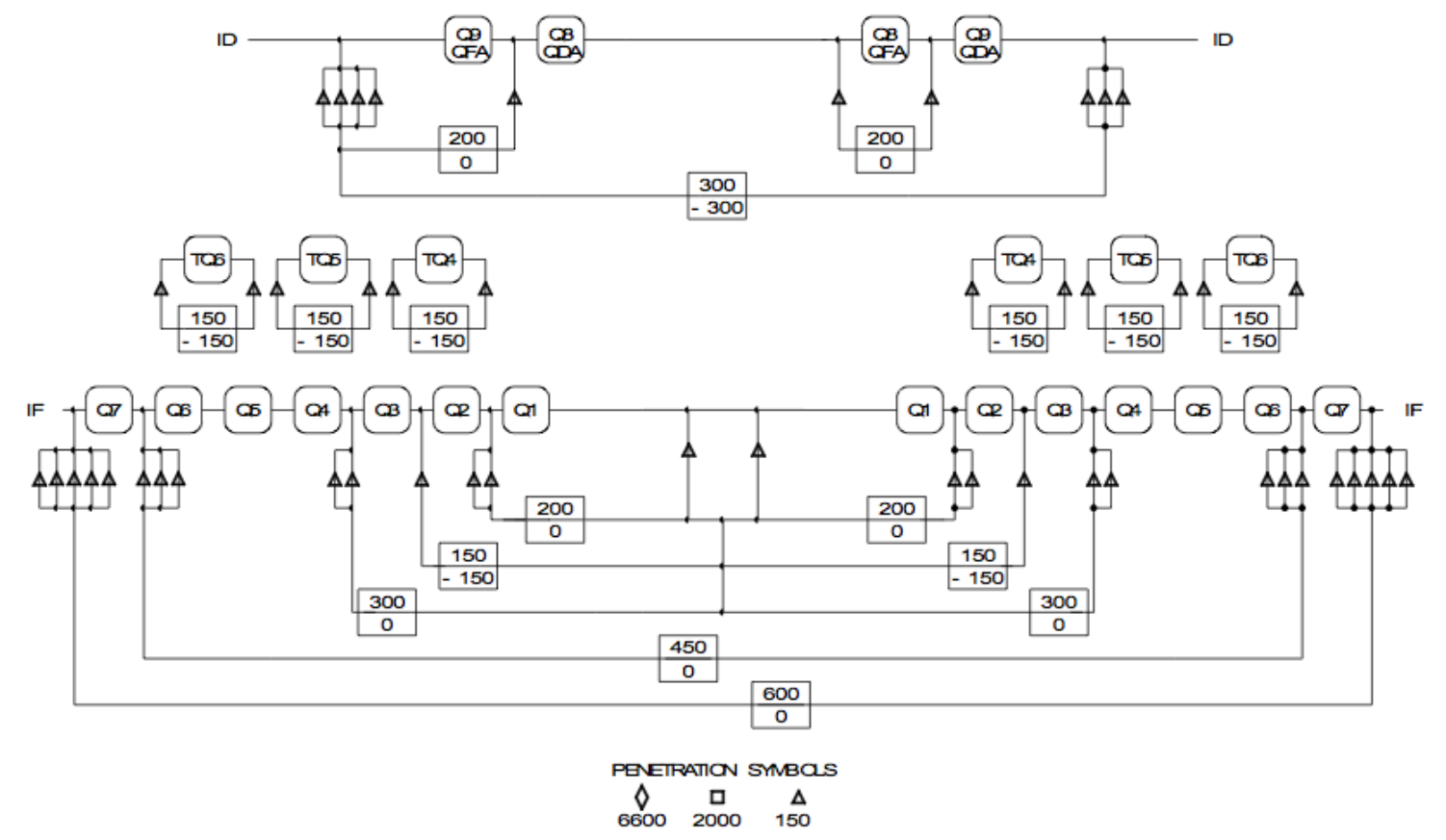

Figure A1: Insertion quads at 2, 6, 8, and 12 o'clock [3]. Independent quadrupoles in the above IRs are: $\mathrm{Q}(1-3), \mathrm{TQ}(4-6), \mathrm{Q} 9$-focusing, and Q8-focusing.

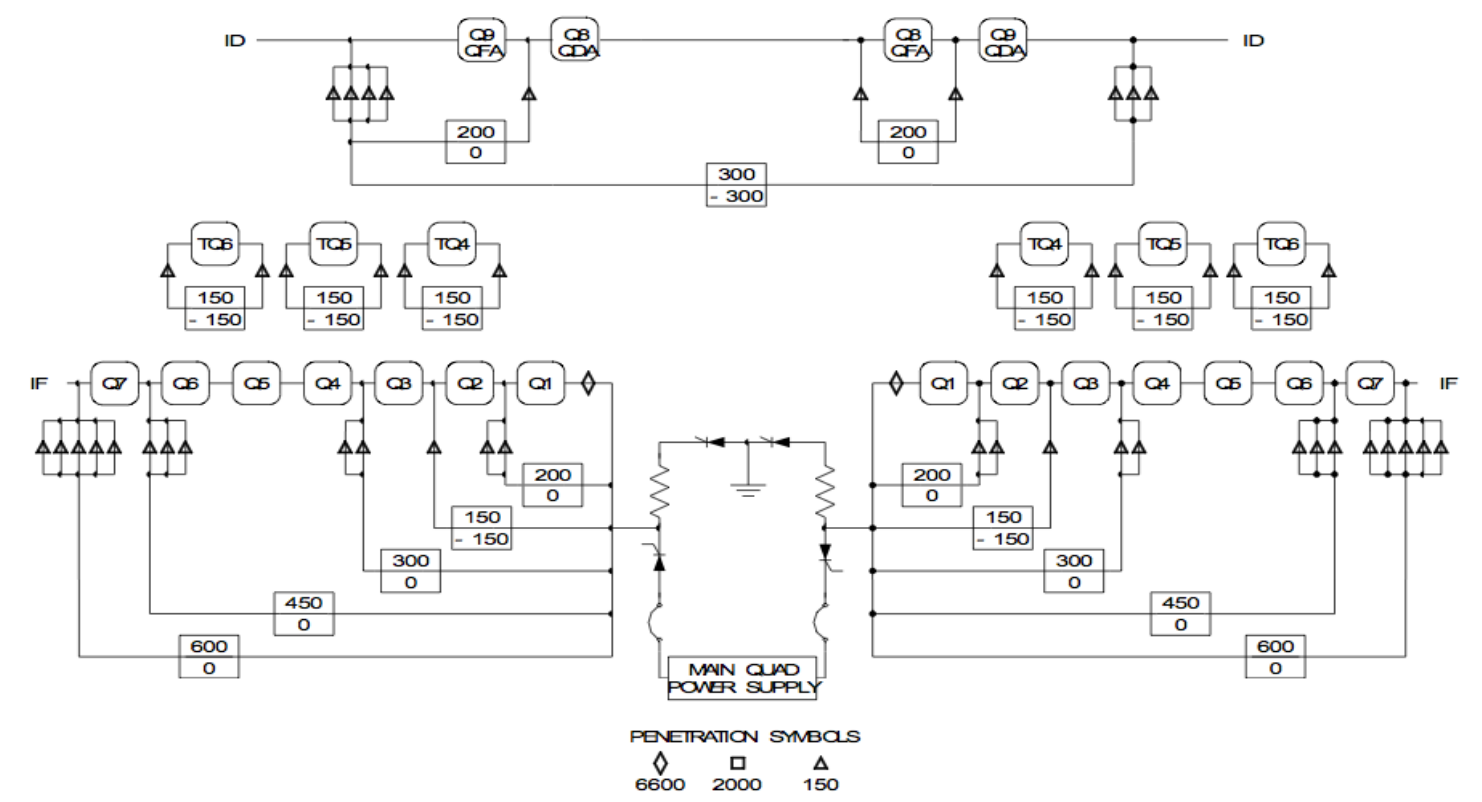

Figure A2: Insertion quadrupoles at IR4 o'clock [3]. Independent quadrupoles in IR4 are: Q(1-3), Q7, TQ(4-6), Q9-focusing, and Q8-focusing. 


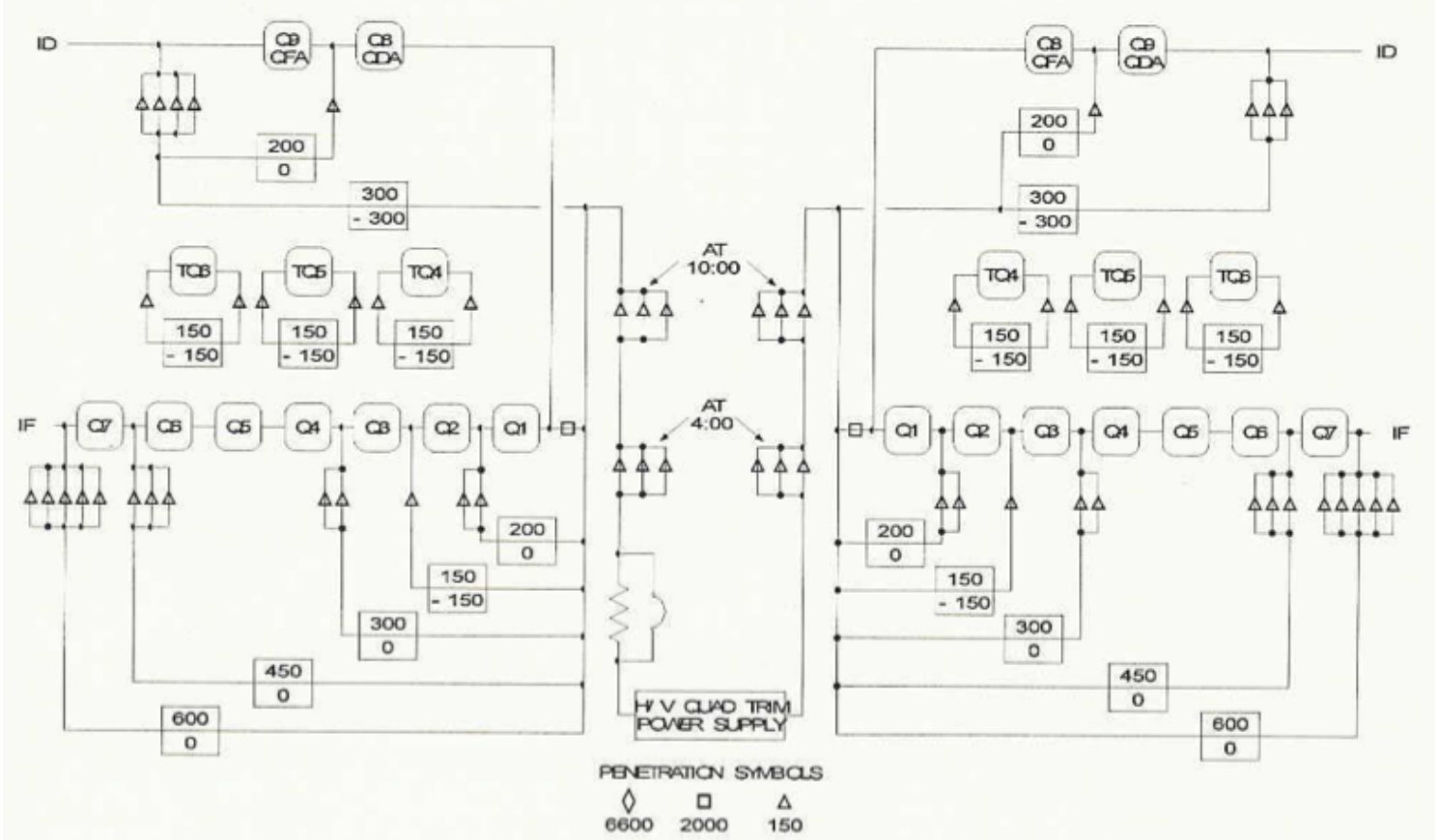

Figure A3: Insertion quadrupoles at 10 o'clock [3]. Independent quadrupoles in IR4 are: $\mathrm{Q}(1-3), \mathrm{Q} 7, \mathrm{TQ}(4-6), \mathrm{Q} 8$, and $\mathrm{Q9}$. 


\section{REFERENCES}

[1] M.G. Minty and F. Zimmermann, "Measurement and Control of Charged Particle Beams", Springer-Verlag, 2003.

[2] P. Rojsel, "A Beam Position Measurement System Using Quadrupole Magnets Magnetic Centra as the Position Reference", Nuclear Instruments and Methods in Physics Research, A 343 p.374-382 (1994).

[3] RHIC Design Manual, BNL, 1993.

[4] J. Niedziela, C. Montag, and T. Satogata, "Quadrupole Beam-Based Alignment at RHIC", Proceedings of PAC'05, p. 3493 (2005); http://www.JACoW.org

[5] J.M. Ziegler and T. Satogata, "Quadrupole Beam-Based Alignment in the RHIC Interaction Regions", Proceedings of PAC'11, p.498 (2011); http://www.JACow.org

[6] J. Beebe-Wang and J.M. Ziegler, "Recent Development on Beam-Based Alignment in RHIC", Proceedings of IPAC'13, p.3082 (2013); http://www.JACoW.org 